\title{
UM ESTUDO SOBRE A CULTURA ORGANIZACIONAL NO SETOR DE COSMÉTICO BRASILEIRO
}

Data de submissão: $11 / 12 / 2014$ Aceite: $22 / 04 / 2017$

André Luiz Romano ${ }^{1}$ Isabela Tatiana Teixeira² Alceu Gomes Alves Filho ${ }^{3}$

André Luís Helleno ${ }^{4}$

\section{RESUMO}

O conceito de cultura pode ser definido como o jeito no qual as coisas são realizadas em determinado ambiente, que vai desde uma casa, até um país, e passa pelos diversos agentes envolvidos nas relações. Para se tratar da questão nas empresas, existe a chamada cultura organizacional. Nas organizações a cultura é influenciada por questões como o poder, a hierarquia e o conservadorismo. A pesquisa apresenta os resultados da avaliação da cultura organizacional no setor de cosmético brasileiro, por meio da análise de segmentação e de correspondência. As análises mostram que o perfil geral da cultura nessas empresas é rígido, e há influência baseada no cargo, priorização dos aspectos individuais, e valores tradicionalmente masculinos. A análise de correspondência mostrou que empresas inglesas e com mais de 500 funcionários apresentam associação com aquelas de maior flexibilidade na cultura, não existência de privilégios diferenciados para níveis distintos, a priorização do grupo em detrimento do individual, acesso dos funcionários aos diretores é comum, e a não presença de divisões de papéis ligados ao gênero. Empresas de nacionalidade chinesa e japonesa associam-se a empresas com divisão clara dos papéis entre homens e mulheres e empresas com ausência de pensamento analítico.

Palavras-chave: Cultura Organizacional; Poder; Setor de Cosmético.

1 Possui graduação em Ciências Econômicas pela Universidade de Araraquara, UNIARA, graduação em Administração pela Pontifícia Universidade Católica de Minas Gerais, PUC Minas, mestrado em Desenvolvimento Regional e Meio Ambiente pela UNIARA, doutorado em Engenharia de Produção pela Universidade Metodista de Piracicaba, UNIMEP e doutorado em andamento em Sustentabilidade Social e Desenvolvimento pela Universidade Aberta de Lisboa, UAb, Portugal. Lisboa - Portugal.

E-mail: andreromano1973@hotmail.com

2 Possui graduação em Ciências Econômicas pela Universidade Estadual Paulista Júlio de Mesquita Filho, UNESP, mestrado em Engenharia de Produção pela Universidade Federal de São Carlos, UFSCAR e doutorado em andamento em Engenharia de Produção pela UFSCAR.

E-mail: isabelatteixeira@hotmail.com

3 Possui graduação em Engenharia de Produção pela Universidade de São Paulo, USP, mestrado em Engenharia de Produção pela USP e doutorado em Engenharia de Produção pela USP. São Carlos - SP. Brasil. E-mail: alceu@ufscar.br

4 Possui graduação em Engenharia de Produção Mecânica pela Universidade Metodista de Piracicaba, UNIMEP, mestrado em Engenharia de Produção pela UNIMEP e doutorado em Engenharia de Produção pela UNIMEP com período sanduíche em Technische Universität Darmstadt. Santa Bárbara d'Oeste - SP. Brasil E-mail: ahelleno@unimep.br 


\section{INTRODUÇÃO}

O processo de intensificação no uso de tecnologias leva a alterações nas atividades das empresas, e cada vez mais segmentos demandam das organizações uma maior capacidade de se apropriar adequadamente de mudanças. Em meio a esse ambiente, os negócios precisam cada vez mais gerenciados com responsabilidade e um nível adequado de visão estratégica, conforme apontado por Milani, Righi e Ceretta (2012). Aspectos como a cultura de determinado ambiente se tornam decisivos ao sucesso dessas mudanças. Segundo Chiavenato (2010) a cultura organizacional é um conjunto de hábitos e crenças estabelecidos por normas, valores, atitudes e expectativas, divididos pelos membros de uma organização; refere-se a um grupo de significados compartilhados por todos os membros e que distingue uma organização das demais.

A ideia de cultura organizacional surgiu entre as décadas de 1970 e 1980 (HOFSTEDE, 1981; OUCHI; PRICE, 1993; SCHWARTZ; DAVIS, 1981; SCHEIN, 1992) como um dos mais controversos temas em gestão (CRANE, 1995). Desde o início houve uma ampla gama de interpretações que resultou numa falta de consenso: entre as definições de cultura incluem-se noções de comportamento, normas e rituais proposto por Trice e Beyer (1984), valores, ideologias e crenças sugeridos por Schwartz e Davis (1981) e formas compartilhadas de significado, como definido por Smircich (1983). Schein (1992) define pressupostos básicos, que constituem o núcleo de uma cultura de determinado grupo. Estes pressupostos ou paradigmas são as maneiras de sentir e perceber o ambiente no entorno de um determinado grupo.

Schein (1992) afirma que a cultura pode ser separada em três níveis, que correspondem ao grau de visibilidade dos fenômenos organizacionais: artefatos; valores; e pressupostos básicos. Schein (2004) aponta esse terceiro nível, como o mais importante para a efetividade de uma mudança organizacional, envolvendo pressupostos básicos de um grupo, que serão tratados na revisão bibliográfica com maior detalhamento. Fleury e Fleury (1997) propõem a separação da cultura organizacional em três níveis: produtos e comportamentos visíveis; histórias, mitos e heróis; e valores. Segundo os autores, esses níveis apresentam uma relação direta com a proposta de Schein, existe uma contribuição importante deles, que é considerar a dimensão política no modelo.

O artigo está estruturado na seção 1 com a apresentação da pesquisa, na seção 2 estão as principais componentes da revisão bibliográfica, e na seção 3 o estudo no setor e principais resultados do survey realizado. $\mathrm{O}$ artigo encerra-se com uma discussão sobre os resultados e as considerações finais.

\section{REVISÃO BIBLIOGRÁFICA}

A cultura organizacional se forma por intermédio da vivência e experiência do grupo em lidar com problemas, sejam eles dentro do processo de aprendizagem segundo o qual se resolvem problemas ou não. Hofstede (1998), Belmonte e Freitas (2013) apontam cultura como uma característica coletiva e não individual. Fleury e Fleury (1997) afirmam que a cultura se refere aos valores profundos de uma organização, desenvolvidos e assimilados pelo grupo ao longo da sua história, e é a forma como uma organização aprendeu a lidar com os problemas (CRANE, 1995), (LINNENLUECKE; RUSSELL; GRIFFITHS, 2009), (ROBBINS; JUDGE, 2013). Schein (1992) propõe um modelo teórico de cultura organizacional composto por três níveis ou dimensões, conforme apresentado na figura 1. 


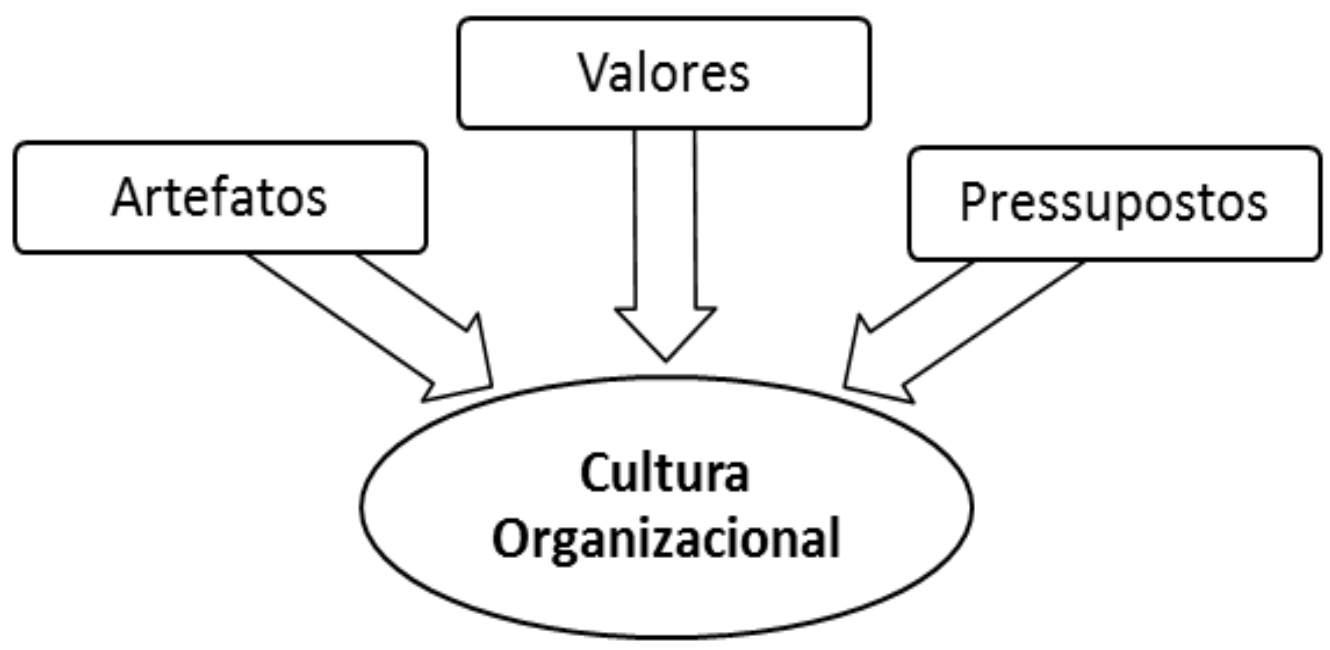

Figura 1 - Componentes da cultura organizacional

Fonte: Baseado em Schein (2004).

Esses três níveis correspondem ao grau de visibilidade que os fenômenos organizacionais apresentam: i) artefatos - incluem os elementos tangíveis, identificáveis de maneira simples numa organização (arquitetura, mobília, código de vestimenta). Trata-se dos elementos visíveis de uma cultura e podem ser reconhecidos por pessoas fora dessa cultura; ii) valores - são os valores declarados por uma organização, como as regras de comportamento. Corresponde à forma como os indivíduos apresentam a organização, tanto para si mesmos, quanto para os outros; e iii) pressupostos básicos - é o aspecto com maior profundidade em cultura. São as ações tomadas de maneira normalmente inconsciente e correspondem à essência dessa cultura, constituindo a parte de maior dificuldade na sua identificação.

Uma organização deve zelar objetivamente pelo correto gerenciamento do terceiro nível. Segundo Schein (2004), é o mais importante para a efetividade de uma mudança organizacional, pois envolve pressupostos básicos de um grupo. Os artefatos são os aspectos visíveis, audíveis, manifestações dos pressupostos básicos, como padrões de comportamento, rituais, ambiente físico, histórias e mitos. Podem ser, por exemplo, como os códigos de vestimenta identificados em algumas empresas, que representam a indicação de alguma cultura básica. Os valores compartilhados compreendem outro componente da cultura organizacional, cujas razões expostas explicam por que as coisas são da forma que são, tais como normas internas, códigos de ética, formalização de valores da companhia e outros; Como exemplo, muitas empresas possuem uma a missão declaradamente, informando aos clientes e colaboradores o que a empresa espera, e vinculando elementos essenciais da cultura organizacional em questão.

Fleury e Fleury (1997) afirmam que a averiguação das práticas organizacionais possibilita o desvendamento da cultura de uma organização. Os autores propõem um modelo no qual a cultura organizacional é subdivida em três níveis: i) produtos e comportamentos visíveis; ii) histórias, mitos e heróis; e iii) valores. Esse modelo pode ser visto na figura 2: 


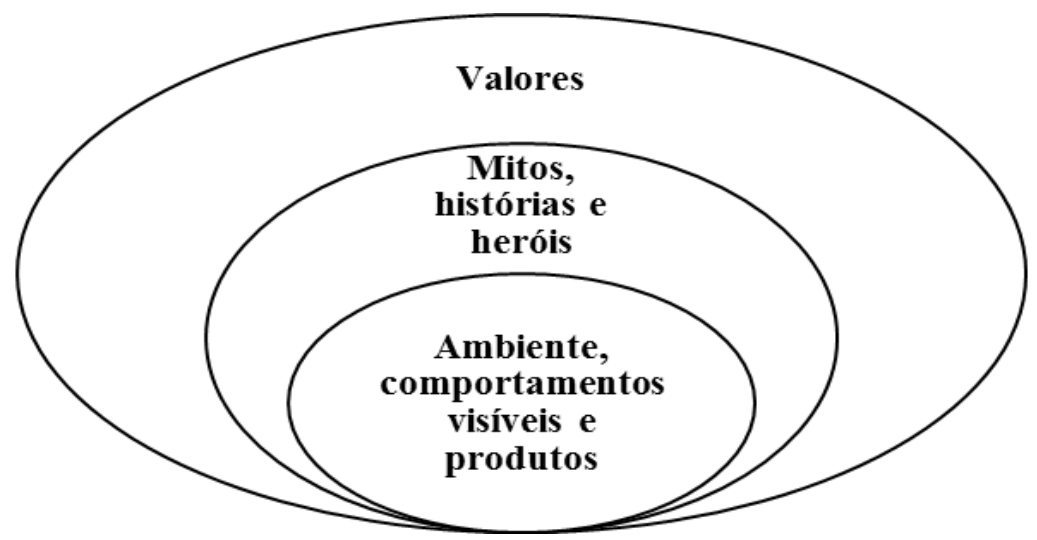

Figura 2 - Os níveis da cultura organizacional

Fonte: Baseado em Fleury e Fleury (1997).

Este modelo apresenta uma relação direta com a proposta originalmente estabelecida por Schein, contendo três níveis, que embora apresentem uma nova denominação, têm um significado próximo ao do modelo original. Adicionalmente, Fleury e Fleury (1997) aprofundam de fato o tema ao adicionarem a dimensão política ao modelo. Segundo eles, a cultura compreende um conjunto de valores definidos por aspectos simbólicos e práticas organizacionais, que atribuem significações para construir uma identidade por meio de sua capacidade de ordenar e gerar sentidos, que podem ser elementos de comunicação e consenso, como apresentado nas relações de dominação.

Com a definição de dado padrão cultural como aceito, passa a ser a forma adequada de se pensar, agir e sentir no grupo. A partir disso, o padrão passa a ser reconhecido como a "forma certa de se fazer as coisas", e qualquer tipo de mudança se torna de difícil realização (FLEURY; FLEURY, 1997). A existência de um padrão cultural definido e aceito é por si só uma resistência a mudanças, sobretudo mudanças que afetem os pressupostos básicos da cultura dessa organização. Segundo Fischer (1989), esses padrões, quando inadequados, podem inviabilizar projetos de mudança que alterem pressupostos básicos, mesmo com todos os argumentos de eficácia ou importância da nova técnica ou modelo de gestão, como no caso da sustentabilidade. Com relação a práticas e valores, Hofstede (1998) afirma que as práticas são seguidas, em geral, por um amplo conjunto de membros da organização, entretanto, nem todos compartilham dos mesmos valores. Separar valores de práticas é recorrentemente visualizado nas organizações, como a questão que envolve a hierarquia, em que um gerente lança mão de seu poder para determinar uma vontade, que nem sempre é a melhor decisão técnica. Tal fato colabora com a afirmação de Hofstede sobre as assimetrias entre práticas e valores dos membros de uma organização.

Baumgartner (2009) apresenta uma abordagem que trata da influência que os programas de sustentabilidade sofrem dos artefatos, valores e pressupostos básicos, que são aspectos culturais de uma organização. Um programa de sustentabilidade afetará as relações de poder dentro da organização, e, sobretudo por se tratar de um conceito novo, é natural que existam resistências de pessoas ou departamentos, que se sentem como se estivessem perdendo seu poder (por exemplo, na condução de um programa de sustentabilidade, as pessoas ou setores que estiverem liderando o projeto terão um maior volume de recursos financeiros à sua disposição, além de maior visibilidade, o que certamente resultará em maior força para impor suas prioridades e objetivos, desencadeando as tais resistências de pessoas ou departamentos).

Denison et al (2012) aponta que um adequado gerenciamento do processo de mudança 
identificará as resistências prováveis e terá que lidar com elas, embora não haja consenso por parte dos autores sobre a melhor forma de lidar com essas resistências. Linnenluecke e Griffiths (2010) apontam que a cultura organizacional é frequentemente tratada como a principal razão do fracasso da adoção de programas de mudança organizacional. As significativas trocas de sistemas e de métodos nas empresas devem ser precedidas pelas mudanças na cultura organizacional instalada. Ao não se trabalhar a cultura do meio organizacional, novas técnicas adotadas podem não ter o sucesso esperado. No momento que não se modificam os pressupostos básicos, as mudanças serão apenas superficiais e a organização estará desperdiçando tempo com ideais não se sustentarão. Para Denison et al (2012) determinados tipos de culturas influenciam decisivamente o sucesso de adoção de novos modelos de negócios. Uma empresa com cultura mais permeável terá maior chance de sucesso, pois terá uma maior disseminação e absorção do que uma cultura fechada e conservadora.

\section{A pesquisa quantitativa para mensuração da cultura}

A cultura organizacional pode ser mensurada pelas dimensões identificadas por Hofstede (1980) e Alcântara et al. (2010) em pesquisas multiculturais. Silva, Medeiros e Enders (2011) apontam que a uma abordagem quantitativa possibilita a descrição da cultura de um ponto de vista do indivíduo externo à organização. Homburg e Pflesser (2000) demonstram que o uso de tipologias quantitativas possibilita uma maior compreensão do fenômeno, ao passo que Schein (1992) considera que podem apresentar um risco de reducionismo que pode prejudicar a compreensão da complexidade da cultura. A abordagem quantitativa é a descrita por Cameron e Quinn (2006) baseada em valores competitivos, com a tipificação de cultura conforme quadro 1.

\begin{tabular}{|c|c|}
\hline Tipologia & Características da Cultura \\
\hline Clã & $\begin{array}{l}\text { É um local de trabalho amigável, no qual as pessoas compartilham experiências tan- } \\
\text { to pessoais como profissionais. A organização é vista como uma extensão da família } \\
\text { e se mantém integrada na base da lealdade e da tradição. A organização valoriza o } \\
\text { trabalho em equipe, a participação, o consenso, a coesão e a moral. }\end{array}$ \\
\hline Hierárquica & $\begin{array}{l}\text { Possui como principal aspecto um local de trabalho formal e estruturado, com regras } \\
\text { e políticas que mantêm a organização integrada e enfatizam a estabilidade, eficiência } \\
\text { e previsibilidade. }\end{array}$ \\
\hline Inovadora & $\begin{array}{l}\text { Direcionam para mudanças e novos desafios, acreditando que o sucesso está na pro- } \\
\text { dução de serviços e produtos únicos e originais. O comprometimento com a experi- } \\
\text { mentação e com a inovação é o que mantém a organização coesa. }\end{array}$ \\
\hline Mercado & $\begin{array}{l}\text { A principal tarefa é guiar a organização em direção à produtividade, dos resulta- } \\
\text { dos e dos rendimentos. O principal negócio da organização é melhorar sua posição } \\
\text { competitiva, assumindo que um propósito claro e uma estratégia agressiva levam a } \\
\text { organização à produtividade e à rentabilidade. }\end{array}$ \\
\hline
\end{tabular}

Quadro 1 - Modelo dos quadrantes de cultura organizacional.

Fonte: Adaptado de Hofstede (1980); Alcântara et al. (2010); Cameron e Quinn (2006).

Os autores apontam que os valores de uma dada organização competem entre si, e isso ocorre devido a divergências e dilemas do sistema organizacional. A cultura é mensurada nas dimensões e valores compartilhados. O modelo incorpora duas principais dimensões: uma baseia-se na estrutura organizacional, com foco na flexibilidade ou controle, e a segunda dimensão é baseada no aspecto organizacional, de acordo com a orientação, ambiente interno ou exter- 
no. Segundo Cameron e Quinn (2006), essas dimensões originam quatro elementos culturais distintos, conforme características e valores assumidos: clã, hierárquica, inovadora e mercado, descrito no modelo gráfico na figura 3. Esse modelo destaca como as culturas podem apresentar diferentes formas de se manifestar.

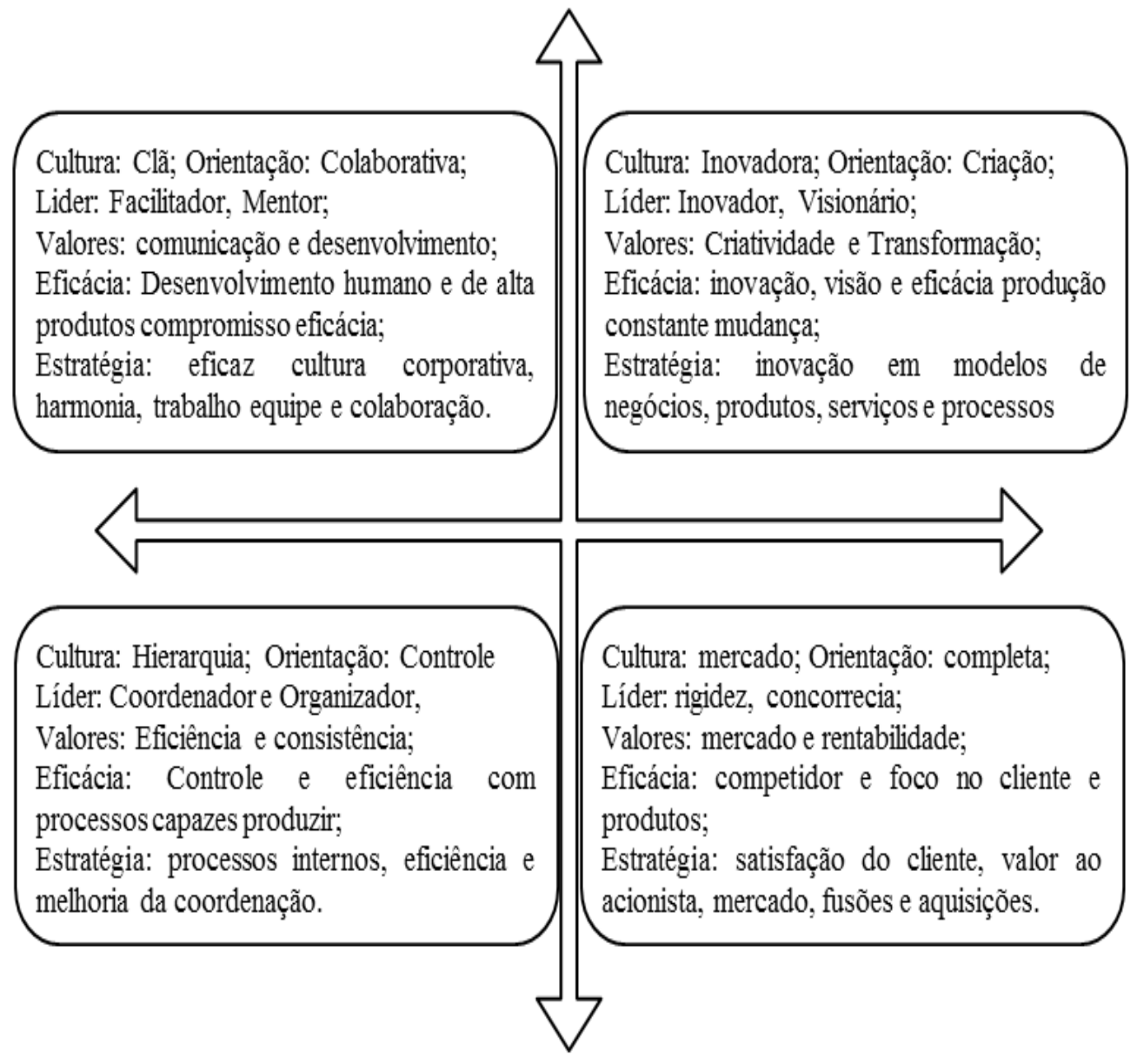

Figura 3 - Os valores competitivos assumidos - elementos culturais Fonte: Baseado em Cameron e Quinn (2006).

O quadro é composto por dois eixos que se cruzam: um deles aborda controle ou flexibilidade e o outro está associado a aspectos internos ou externos à organização. Quando se enfatiza o controle, existe a confiança em mecanismos formais, como regras, políticas e orçamentos para o cumprimento das normas de comportamento. Quando esse foco está na flexibilidade, existe maior confiança na internalização de crenças, formação, compartilhamento e pressão do próprio grupo para atendimento dos resultados e comportamentos desejados. Esse quadro resulta em quatro quadrantes que correspondem a diferentes tipos de culturas:

superior esquerdo - são culturas dominadas por valores humanos, que pretendem promover a coesão moral entre os colaboradores. É possível desenvolver os recursos humanos, criar um processo de comunicação interna eficiente, além de se ter um ambiente participativo para a tomada de decisão. Nesse quadrante, existe a necessidade de obediência individual aos resultados organizacionais, baseados em tradição, confiança e compromisso de longo prazo. Técnicas como o trabalho em equipe, colaboração, capacitação, gestão de talentos, ou as relações inter- 
pessoais estão associadas a esse quadrante;

superior direito - são culturas dominadas por valores de sistemas abertos, que apresentam foco no crescimento e aquisição de recursos através da promoção da mudança, adaptabilidade e prontidão, comunicação visionária, flexível e tomada de decisão. A coordenação apresenta uma característica mais informal. O que motiva os individuos é o significado ideológico. São técnicas desse quadrante as focadas em inovação, criatividade, mudança, transformação ou empreendedorismo;

inferior esquerdo - são culturas dominadas por valores de processo internos, que buscam promover a estabilidade e controle com meios formais, como gestão da informação. Esse tipo de cultura também tem sido chamado de cultura hierárquica, pois envolve a conformidade, aplicação das regras e atenção a questões técnicas. Coordenação e controle são realizados através de comunicação vertical, políticas e procedimentos; conta também com regras e regulamentos. São técnicas associadas a esse quadrante a avaliação e medição, controle de processos, estruturação, melhoria e eficiência da qualidade;

inferior direito - são dominadas por valores e metas racionais, que promovem a eficiência e produtividade. Trata-se de uma abordagem racional de cultura com tomada de decisão centralizada. Os indivíduos são motivados por uma crença de que serão recompensadas pela sua competência no desempenho das metas organizacionais. Compõe esse quadrante as técnicas com foco na competitividade, a resposta rápida, capacidade de vencer barreiras, metas e realizações.

Ao se efetuar uma averiguação sobre essas tipologias de cultura, verifica-se que não são mutuamente excludentes, embora predominâncias de determinadas tipologias sejam esperadas. Esse modelo apresenta um importante papel na determinação de priorização de um equilibrio entre a estabilidade e adaptação, determinando a priorização do direcionamento entre demandas concorrentes. Quinn (2000) aponta que organizações com foco excessivo num tipo de cultura estarão mais arriscadas a se tornarem disfuncionais. Por exemplo, uma orientação para valores de processos internos, localizada no quadrante inferior esquerdo, resultará numa burocracia rígida, resistente a iniciativas de mudança. Como a sustentabilidade compreende mudanças que têm por objetivo mexer com as estruturas e valores internos, é preciso que as culturas apresentem um amadurecimento dessas características para que a sustentabilidade tenha sucesso.

Outra tipologia apontada é de Hofstede (1980), que realizou comparações entre culturas nacionais e em empresas multinacionais em vários países, detalhando cinco dimensões: distância hierárquica, individualismo, masculinidade, controle da incerteza e visão de curto e longo prazo. Alcântara, Reis e Meneses (2012) utilizaram o modelo de Hofstede, por meio de estatísticas multivariadas para avaliação da cultura organizacional. As dimensões de Hofstede (1980) são:

Índice de Distância Hierárquico (IDH) é uma medida na qual os colaboradores com menor poder aceitam uma assimetria na distribuição de poder. Nesses casos é provável que exista uma definição bem estabelecida, com dificuldade na mobilidade entre os níveis. Alcântara et al. (2010) afirma que uma menor distância hierárquica é um indicativo de não haver importância entre as diferenças dos membros por poder e riqueza;

O Índice de Individualismo (INDI) é a medida que uma organização se baseia no individual, coletivo e relações interpessoais. Um alto nível de individualismo aponta priorização dos direitos individuais, num ambiente no qual os participantes tendem a formar um maior número de relações fora do ambiente de trabalho; já num ambiente com baixo individualismo, a organização apresenta-se coletivista, com laços entre os colaboradores. Sociedades coletivas são caracterizadas por Motta e Caldas (1997) e Alcântara et al. (2010) por relações mais profundas, e as 
pessoas distinguem grupos com interesses similares, e há proteção entre os membros e troca de lealdade;

Índice de Masculinidade (IMASC) é o grau com que se reforçam valores tradicionalmente masculinos de realização, como o controle e o poder. Para empresas com um baixo índice de masculinidade, pode-se concluir que existe um baixo índice de diferenciação entre gêneros. São valores da sociedade masculina a assertividade e o enriquecimento, não apresentando ênfase em pessoas. São características de organizações com cultura de baixo índice de IMASC valores baseados em relacionamentos, pessoas e na qualidade de vida;

Índice de Controle da Incerteza (ICI) está presente em organizações em que se busca evitar as surpresas e, por conta disso, as incertezas. Em organizações com um menor foco em evitar incertezas, existe tolerância à multiplicidade de opiniões e também uma menor orientação para regras; são mais flexíveis a mudanças e dispostos a assumirem maiores riscos (HOFSTEDE, 1980);

Índice da Visão em longo prazo ou curto prazo (IVI) demonstra quando uma organização se baseia em tradições relativas a acontecimentos passados e presentes ou sobre os benefícios do que é desejável ao futuro. No longo prazo os valores serão orientados para o futuro, como poupanças e persistência. Por outro lado, numa visão de curto prazo, os valores têm uma orientação para o passado e o presente, aceitando como verdade a tradição e o cumprimento das obrigações sociais.

As dimensões de Hofstede permitem identificar a cultura predominante, dadas as características do sistema, se rígido ou flexível, conforme o quadro 2.

\begin{tabular}{|c|c|c|}
\hline $\begin{array}{l}\stackrel{U}{d} \\
. \frac{0}{0} \\
.\end{array}$ & $\begin{array}{c}\text { Características de Sistema } \\
\text { Flexível }\end{array}$ & $\begin{array}{c}\text { Características de Sistema } \\
\text { Rígido }\end{array}$ \\
\hline II & $\begin{array}{l}\text { Pequena distância hierárquica } \\
\text { Desigualdades devem ser mínimas; } \\
\text { Interdependência dos níveis de poder; } \\
\text { Hierarquia é forma de desigualdade; } \\
\text { A descentralização é comum; } \\
\text { Diferença salarial mínima entre níveis; } \\
\text { Subordinados esperam ser consultados; } \\
\text { O chefe ideal é democrata e competente; } \\
\text { Privilégios e "status" são mal vistos. }\end{array}$ & $\begin{array}{c}\text { Grande distância hierárquica } \\
\text { Desigualdades são desejáveis. } \\
\text { Dependência dos níveis de poder; } \\
\text { Hierarquia é desigualdade existencial; } \\
\text { A centralização é comum; } \\
\text { Diferença salarial elevada entre níveis; } \\
\text { Subordinados esperam ser orientados; } \\
\text { O chefe ideal é autocrata benevolente; } \\
\text { Os privilégios “status" são essenciais... }\end{array}$ \\
\hline$\underline{\underline{\Omega}}$ & $\begin{array}{c}\text { Sociedades coletivas } \\
\text { Grupos se cuidam entre si, lealdade e troca; } \\
\text { A identidade é função do grupo social; } \\
\text { Harmonia, evitando confrontos diretos; } \\
\text { Emprego em condições morais; } \\
\text { Decisões consideram grupos empregados; } \\
\text { Administrar relacionamentos; } \\
\text { A relação prevalece sobre a tarefa. }\end{array}$ & $\begin{array}{l}\text { Sociedades individualistas } \\
\text { Pessoa cresce, e cuida de si e dos seus; } \\
\text { A identidade baseada no individuo; } \\
\text { Dizer o que pensa é para os honestos; } \\
\text { Emprego em condições de vantagens; } \\
\text { Decisões de acordo com as regras; } \\
\text { Administração a tarefa; } \\
\text { A tarefa prevalece sobre a relação. }\end{array}$ \\
\hline$\stackrel{u}{\underline{4}}$ & $\begin{array}{c}\text { Sociedades femininas } \\
\text { Valores: atenção e cuidado pelos outros; } \\
\text { Homens e mulheres podem ser ternos; } \\
\text { Trabalhar para viver; } \\
\text { Intuição e a qualidade de vida no trabalho; } \\
\text { Igualdade, solidariedade, qualidade de vida; } \\
\text { Conflitos resolvidos com negociação; } \\
\text { Todos devem ser modestos; } \\
\text { Importância de pessoas, relações calorosas. }\end{array}$ & $\begin{array}{c}\text { Sociedades masculinas } \\
\text { Valores: sucesso e progresso material; } \\
\text { Mulheres são ternas e relacionam-se; } \\
\text { Viver para trabalhar; } \\
\text { Decisivos e auto afirmativos; } \\
\text { Equidade, competição e desempenho; } \\
\text { Conflitos resolvidos por confronto; } \\
\text { Autoconfiança, ambição e dureza; } \\
\text { A importância do dinheiro e bens. }\end{array}$ \\
\hline
\end{tabular}




\begin{tabular}{|c|c|c|}
\hline$\overline{\underline{z}}$ & $\begin{array}{c}\text { Baixo controle da incerteza } \\
\text { Pouco stress, bem-estar; } \\
\text { Emoção e agressão devem ser minimizadas; } \\
\text { Educação flexível ao que é sujo e tabu; } \\
\text { O que é diferente é curioso; } \\
\text { Existir o menor número possível de regras; } \\
\text { O tempo é apenas marco de referência; } \\
\text { Trabalha-se muito quando é necessário; } \\
\text { Precisão e a pontualidade são aprendidas; } \\
\text { Tolerância e moderação prevalecem. }\end{array}$ & $\begin{array}{c}\text { Elevado controle da incerteza } \\
\text { Stress elevado, ansiedade; } \\
\text { Emoções e agressão podem ocorrer; } \\
\text { Normas estritas sobre tabu e sujo; } \\
\text { O que é diferente é perigoso; } \\
\text { Demanda regras, mesmo ineficazes; } \\
\text { Tempo é dinheiro. } \\
\text { Necessidade emocional de ocupação; } \\
\text { Precisão e pontualidade são naturais; } \\
\text { Resistência para inovação. }\end{array}$ \\
\hline$\geq$ & $\begin{array}{l}\text { Visão de Curto Prazo } \\
\text { Valores: liberdade, respeito aos direitos. } \\
\text { Devem produzir resultados rápidos. } \\
\text { Foco na geração dos lucros deste ano. } \\
\text { Gestores e funcionários são diferentes. } \\
\text { Recompensa: meritocracia, por habilidades. } \\
\text { Lealdade atende demandas do negócio. } \\
\text { A preocupação em possuir a Verdade. } \\
\text { Pensamento analítico. }\end{array}$ & $\begin{array}{l}\text { Visão de Longo Prazo } \\
\text { Valores: honestidade, autodisciplina e responsabil- } \\
\text { idade. } \\
\text { O foco está em crescer, ganhar mercado. } \\
\text { Importância dos lucros daqui a dez anos. } \\
\text { Todos compartilham mesmas aspirações. } \\
\text { Satisfação em corrigir injustiças. } \\
\text { A prioridade é dada ao bem comum. } \\
\text { Desacordo não faz mal. } \\
\text { Pensamento sintético. }\end{array}$ \\
\hline
\end{tabular}

Quadro 2 - Organizações baseadas na flexibilidade ou no controle.

Fonte: Adaptado de Hofstede (1980); Alcântara et al. (2012).

Hofstede (1980) afirma serem as dimensões culturais representações importantes da possibilidade para desenvolvimento de pesquisas organizacionais. Essas pesquisas têm sido utilizadas para descrever a percepção de valores culturais entre países, grupos de uma mesma organização ou entre organizações. Foram encontrados outros autores cujas pesquisas colaboram para o desenvolvimento do tema, entre os quais se encontra Souza (1978) e Ramos e Cardoso (1989), que possuem grande relevância.

Uma abordagem quantitativa fixa alguns elementos culturais universais, permitindo análises comparativas entre diferentes organizações ou grupos menores, em subculturas. De acordo com essa abordagem, é possível também analisar relações entre as variáveis e investigar sistemas longitudinais, estáveis ao longo do tempo. Hofstede (1991) aponta para a maior credibilidade dos estudos quantitativos devido à maior independência, imparcialidade e neutralidade do pesquisador. $\mathrm{O}$ atual estado da arte das técnicas e softwares estatísticos auxilia este aumento de confiabilidade, precisão e objetividade. Para Fleury e Fleury (1997) é necessário ter cuidados na construção das hipóteses, elaboração da coleta de dados, validação, no desenho da amostra e utilização de ferramentas estatísticas aderentes ao tipo de dado coletado, evitando com isso resultados sem validade e confiabilidade. Santos (2000) aponta que métodos quantitativos se apresentam como uma ferramenta mais rápida para a coleta e interpretação de dados e apresentação dos resultados. Fleury e Fleury (1997) afirmam que os números permitem reforço nas análises e recomendações dos pesquisadores.

A maior crítica ao modelo quantitativo vem de Schein (1992), figura eminente e desenvolvedora de parte da teoria da Cultura Organizacional, juntamente com Hofstede; seu argumento é que a cultura é abstrata e medi-la é impossível. Segundo ele os instrumentos de coleta de dados identificam apenas alguns artefatos e valores, mas dificilmente revelam os pressupostos básicos. A quantidade de dimensões envolvidas faz, segundo ele, com que um questionário tenha que ser imenso para ser efetivo. Pelo observado na revisão, não existe a possibilidade de se afirmar que uma abordagem qualitativa é superior à quantitativa e vice-versa, pois as técnicas devem ser selecionadas para se adequar ao objeto de estudo. A escolha do método é orientada pelos pressupostos do pesquisador com o problema de pesquisa. É preciso um método que seja coerente com a base teórica utilizada, para interpretar os dados. Existe uma corrente de autores 
que vem sugerindo o uso de abordagens híbridas. A composição de abordagens, denominada triangulação. Segundo Santos (2000), a triangulação é um processo pelo qual são utilizados diferentes métodos de coleta de dados ou instrumentos de pesquisa simultaneamente. Ao combinar métodos é possível uma descrição holística do fenômeno, facilitando a compreensão, podendo revelar algo desconhecido quando aplicado a único método. A triangulação pode proporcionar maior confiabilidade, validade e consistência. Entretanto, sua aplicação não é tão simples e exige mais trabalho e tempo por parte do pesquisador, além de aumentar os custos no orçamento do projeto.

Fleury e Fleury (1997) classificam quatro tipos básicos de triangulação: i) de dados, subdivididos em tempo, espaço e pessoa; ii) do investigador, com diversos observadores para um mesmo objeto; iii) de teoria, que inclui diversas perspectivas teóricas para analisar um mesmo objeto; iv) metodológica, entre métodos - métodos distintos desenvolvem dados comparáveis; e v) intramétodo - técnicas de um mesmo método na coleta de dados.

A discussão sobre cultura permitiu uma ampla caracterização dessa teoria. A cultura apresenta algumas dimensões e elementos gerais dos quais se desdobram questões para a determinação da predominância do tipo de cultura. A hierarquia foi o primeiro elemento identificado, podendo ser também reconhecida como a distância do poder. Para se determinar a hierarquia são utilizados aspectos como, quanto os membros menos poderosos aceitam e esperam distribuição desigual de poder; qual o sistema de valores daqueles que têm menos poder. A segunda dimensão identificada foi o nível de individualismo, que aborda até que ponto as pessoas sentem que podem tomar conta de si próprias, das suas famílias ou das empresas. A terceira dimensão é a flexibilidade da organização, que aborda o nível de machismo incutido na organização. Por exemplo, como a o poder está concentrado nas mãos dos homens, quão agressivo e competitivo é o seu comportamento e o quando negocia, como também se está mais interessado em resultados ou em justiça. Na quarta dimensão está o grau de ameaça que o ambiente impõe a seus indivíduos, podendo refletir um ambiente de desconforto, mediante a sensação de riscos, caos e situações não estruturadas. Adicionalmente, esses sentimentos são expressos por meio de estresse nervoso e uma necessidade de previsibilidade, como regras claras e escritas. A quinta dimensão relaciona-se à capacidade de inovação que determinada cultura tem. É difícil inovar em ambientes que pensem no curto prazo, pois apresentam valores orientados ao passado e o presente como respeito pela tradição e cumprimento de obrigações sociais. Empresas inovadoras que apresentam culturas de longo prazo têm valores orientados para o futuro, como economia (poupança) e persistência. Da caracterização de cada perfil, espera-se que haja um tipo que possa ser favorável para o sucesso da sustentabilidade corporativa.

\section{APLICAÇÃO DE UMA AVERIGUAÇÃO NO SETOR DE COSMÉTIÇO BRASILEIRO}

O segmento de cosmético faz parte do setor denominado Higiene Pessoal, Perfumaria e Cosméticos (HPPC). Segundo a ABIHPEC, esse setor apresentou um crescimento médio de 10,09\% entre 1996 e 2011, e passou de um faturamento líquido de imposto sobre vendas de MMR\$ 4,9 em 1996, para MMR\$29,4 em 2011. Para a ABIHPEC (2012) o crescimento desse setor se deve a fatores como a crescente participação da mulher no mercado de trabalho e a utilização de alta tecnologia. O aumento da produtividade é responsável pelos preços do setor apresentar inflação menor do que os índices gerais da economia. Outro aspecto que contribui para o seu 
crescimento é o aumento da expectativa de vida, que implica na necessidade de conservar uma impressão de juventude. De acordo com a ABIHPEC (2012), há 1.659 empresas no setor de higiene pessoal, perfumaria e cosméticos; dessas, 330 são classificadas pela CosméticosBR (http:// www.cosmeticosbr.com/index.asp) como produtoras e comercializadoras de produtos acabados.

O grupo de produtos acabados foi convidado a participar da presente pesquisa; o questionário foi encaminhado a 330 empresas, as quais retornaram 102 respostas. Os dados foram coletados por meio do questionário on-line do site www.surveymonkey.com.br aplicado entre Maio e Julho de 2013, e parte aplicada via telefone/e-mail pelo pesquisador. O objetivo do questionário foi verificar a percepção sobre cultura organizacional das empresas de cosméticos que atuam no Brasil. Foi utilizada uma escala crescente de concordância de cinco pontos.

A análise de segmentação e a análise de correspondência foram utilizadas para a análise dos dados; a análise de segmentação corresponde a recortes que permitem avaliar a opinião de grupos específicos da amostra; a análise de correspondência é uma estatística do grupo da análise multivariada, se tratando de um método de redução de dados, que objetiva a obtenção de agrupamentos de dados similares entre si, com a finalidade de classificar conforme as relações naturais da amostra, formando grupos de objetos (porte das empresas, nacionalidade, mercado de atuação, entre outras).

\subsection{Análise das questões de Cultura Organizacional}

As questões de cultura foram avaliadas utilizando uma escala crescente de concordância, com a seguinte composição: (1) Nunca; (2) Na minoria das vezes; (3) Algumas vezes; (4) Na maioria das vezes; e (5) Sempre. O primeiro grupo de questões foi o Índice de Distância Hierárquico (IDH), que se trata de uma medida em que os colaboradores com menor poder aceitam uma assimetria nessa distribuição. Um maior distanciamento entre a hierarquia aponta para maiores desigualdades de poder e riqueza. É mais provável que existam nessas empresas uma definição bem estabelecida, com dificuldade na mobilidade entre os níveis. Os dados contidos na tabela 1 abordam aspectos de distância hierárquica.

\begin{tabular}{|c|c|c|c|c|c|c|}
\hline 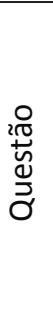 & 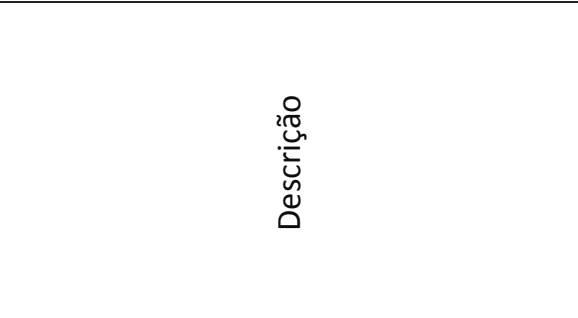 & 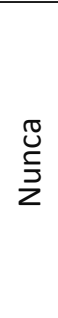 & 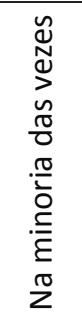 & 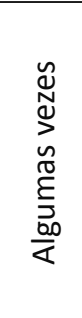 & 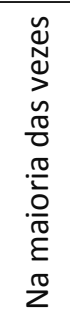 & $\frac{\searrow}{\frac{0}{2}}$ \\
\hline 1 & $\begin{array}{l}\text { A influência de uma pessoa é baseada } \\
\text { primeiramente no cargo que ela ocupa }\end{array}$ & 0,0 & 29,4 & 39,2 & 28,4 & 2,9 \\
\hline 2 & $\begin{array}{l}\text { Os funcionários não possuem liberdade } \\
\text { para questionar as ordens superiores }\end{array}$ & 4,9 & 33,3 & 35,3 & 24,5 & 2,0 \\
\hline 3 & $\begin{array}{l}\text { Os funcionários não podem falar } \\
\text { diretamente com seus diretores }\end{array}$ & 9,8 & 34,3 & 29,4 & 23,5 & 2,9 \\
\hline 4 & $\begin{array}{l}\text { As posições mais altas têm privilégios } \\
\text { diferentes das posições mais baixas }\end{array}$ & 4,9 & 20,6 & 39,2 & 30,4 & 4,9 \\
\hline
\end{tabular}

Tabela 1 - Avaliação do índice de distância hierárquica (IDH) Fonte: Próprio autor. 
As questões de cultura organizacional das empresas avaliadas apontam, de forma geral, que as pessoas têm pelo menos alguma influência baseada primeiramente no cargo que elas ocupam (as respostas se concentraram entre 'Na minoria das vezes', 'Algumas vezes' e 'Na maioria das vezes'). A partir da avaliação desses dados, verifica-se que $68,6 \%$ das empresas afirmam que na minoria das vezes ou alguma vez possuem liberdade para questionar as ordens dos superiores. Além disso, $4,9 \%$ dos respondentes afirmam nunca terem liberdade para questionamento de ordens. Os funcionários das empresas podem falar diretamente com seus diretores pelo menos na minoria das vezes, com $90,2 \%$, ao mesmo tempo em que $9,8 \%$ das empresas afirmam que não há esse diálogo. A maioria dos respondentes afirmou que em alguma ou na maioria das vezes as posições mais elevadas hierarquicamente têm privilégios diferentes daqueles na posição baixa, com $69,6 \%$.

O segundo grupo é relativo ao Índice de Individualismo (INDI), que é a medição do quanto uma organização baseia-se no individual, coletivo e relações interpessoais. Um alto nível de individualismo aponta priorização dos direitos individuais, num ambiente no qual os participantes tendem a formar um maior número de relações fora do ambiente de trabalho. Por outro lado, num ambiente com baixo individualismo, a organização apresenta-se mais coletivista, com laços entre os colaboradores. Conforme mostra a tabela 2, a maior parte das empresas indicou que os funcionários usam algumas vezes individualismo e egoísmo em suas relações profissionais, com $47,1 \%$. Além disso, elas afirmam que o indivíduo é mais valorizado que o grupo e que na empresa não existe trabalho em grupo em algumas vezes e na maioria das vezes $(69,6 \%)$.

\begin{tabular}{|c|c|c|c|c|c|c|}
\hline 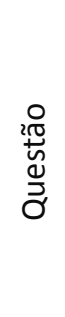 & 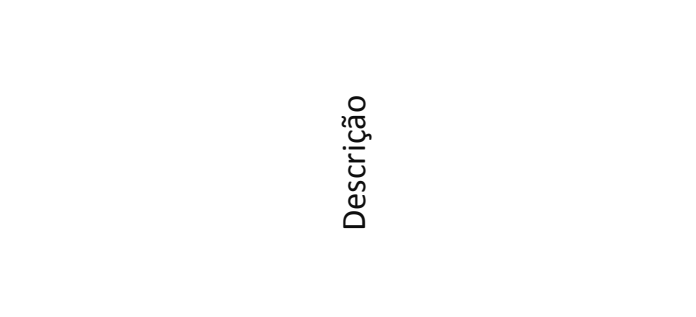 & $\begin{array}{l}\text { 이 } \\
\stackrel{5}{z}\end{array}$ & 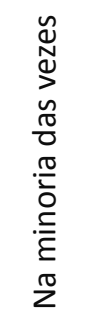 & 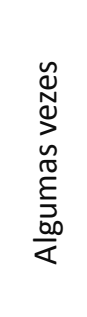 & 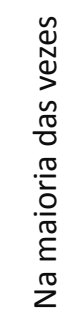 & 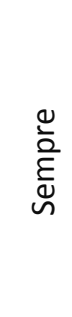 \\
\hline 5 & $\begin{array}{l}\text { Os funcionários usam individualismo e egoísmo } \\
\text { em suas relações profissionais. }\end{array}$ & 3,9 & 24,5 & 47,1 & 21,6 & 2,9 \\
\hline 6 & $\begin{array}{l}\text { O indivíduo é mais valorizado que o grupo, } \\
\text { não existe trabalho em grupo. }\end{array}$ & 8,8 & 19,6 & 39,2 & 30,4 & 2,0 \\
\hline 7 & $\begin{array}{l}\text { Os interesses individuais } \\
\text { sobrepõem os coletivos. }\end{array}$ & 9,8 & 14,7 & 44,1 & 30,4 & 1,0 \\
\hline 8 & $\begin{array}{l}\text { As relaçôes de competição profissional } \\
\text { são claramente percebidas. }\end{array}$ & 2,0 & 19,6 & 45,1 & 32,4 & 1,0 \\
\hline
\end{tabular}

Tabela 2 - Avaliação do índice de individualismo (INDI)

Fonte: Próprio autor.

Consequentemente, os interesses individuais sobrepõem os coletivos, algumas vezes com $44,1 \%$. Outro fator destacado é que existem, em algumas vezes, relações de competição profissional claramente percebida para a maior parte das empresas, com 45,1\%.

O terceiro grupo é o Índice de Masculinidade (IMASC), que corresponde ao grau com que se reforçam valores tradicionalmente masculinos de realização, como o controle e o poder. $\mathrm{O}$ alto peso nesse índice é indicativo de que existe diferenciação de gênero, situação na qual as mulheres são dominadas ou controladas pelo homem. Para empresas com um baixo índice de 
masculinidade, pode-se concluir que existe um baixo índice de diferenciação entre gêneros. Corresponde a distinção de gênero nas organizações, tendo como valores da sociedade masculina a assertividade e o enriquecimento, não apresentando ênfase em pessoas. São características de organizações com cultura com baixo índice de IMASC os valores dominantes baseados em relacionamentos, nas pessoas, no próximo e na qualidade de vida.

Conforme o visto na tabela 3, verifica-se que $42,2 \%$ das empresas respondentes afirmaram que, algumas vezes, a maioria dos funcionários caracteriza-se por indelicadeza. É um indicativo de que a maior parte das empresas participantes tem o individualismo presente algumas vezes no dia a dia. A maioria das empresas $(45,1 \%)$ afirma que os funcionários escondem suas emoções algumas vezes. A maioria das empresas afirma ter poucas ou nenhuma mulher nos cargos gerenciais e que, pelo menos alguma vez ou na maioria das vezes $(65,7 \%)$, ocorre uma divisão clara dos papéis entre homens e mulheres. Neste caso, 33,3\% das empresas dizem nunca ou na minoria das vezes haver essa divisão clara de papéis.

\begin{tabular}{|c|c|c|c|c|c|c|}
\hline 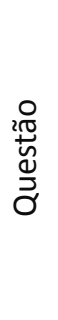 & 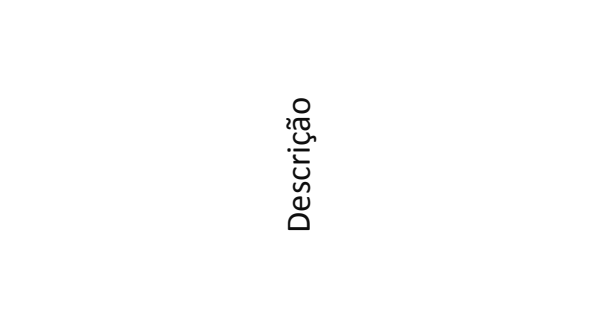 & 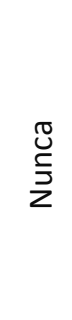 & 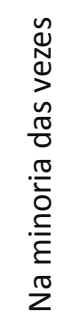 & 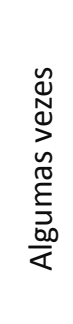 & 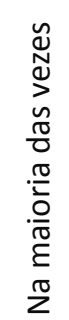 & 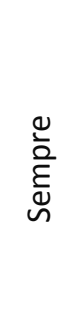 \\
\hline 9 & $\begin{array}{l}\text { A maioria dos funcionários } \\
\text { caracteriza-se por indelicadeza. }\end{array}$ & 11,8 & 27,5 & 42,2 & 17,6 & 1,0 \\
\hline 10 & $\begin{array}{l}\text { Existem poucas ou nenhuma mulher } \\
\text { em cargos gerenciais ou administrativos. }\end{array}$ & 5,9 & 25,5 & 40,2 & 28,4 & 0,0 \\
\hline 11 & $\begin{array}{l}\text { A maioria dos funcionários } \\
\text { esconde as suas emoções. }\end{array}$ & 1,0 & 24,5 & 45,1 & 28,4 & 1,0 \\
\hline 12 & $\begin{array}{l}\text { Ocorre uma divisão clara dos papéis } \\
\text { entre homens e mulheres. }\end{array}$ & 13,7 & 19,6 & 39,2 & 26,5 & 1,0 \\
\hline
\end{tabular}

Tabela 3 - Avaliação do índice de masculinidade (IMASC)

Fonte: Elaborado pelo autor

O quarto grupo é o Índice de Controle da Incerteza (ICI), que está presente em organizações nas quais se busca evitar as surpresas e, por conta disso, as incertezas. Apresenta baixa tolerância à ambiguidade e é orientada por normas, regras ou controles, reduzindo o nível de incerteza. Em organizações com um menor foco em evitar incertezas, existe tolerância à multiplicidade de opiniões e também uma menor orientação para regras. São mais flexíveis às mudanças e dispostos a assumirem riscos. Avalia-se esse aspecto conforme a tabela 4. 


\begin{tabular}{|c|c|c|c|c|c|c|}
\hline 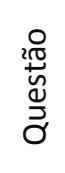 & 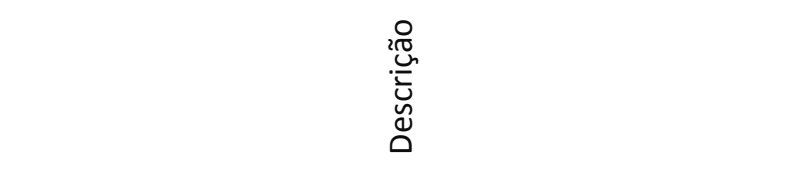 & 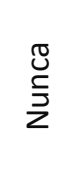 & 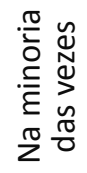 & 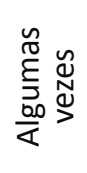 & 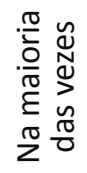 & 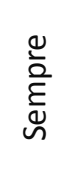 \\
\hline 13 & $\begin{array}{l}\text { Os funcionários quase nunca se sentem } \\
\text { tensos ou nervosos. }\end{array}$ & 1,0 & 22,5 & 49,0 & 24,5 & 2,9 \\
\hline 14 & $\begin{array}{l}\text { A maioria dos funcionários prefere tarefas } \\
\text { bem estruturadas e claras. }\end{array}$ & 2,0 & 15,7 & 38,2 & 35,3 & 8,8 \\
\hline 15 & $\begin{array}{l}\text { Grande parte do trabalho é planejada, } \\
\text { levando a poucos eventos inesperados. }\end{array}$ & 2,0 & 19,6 & 42,2 & 35,3 & 1,0 \\
\hline 16 & $\begin{array}{l}\text { As reuniỗes frequentemente são planejadas com ante- } \\
\text { cedência. }\end{array}$ & 1,0 & 24,5 & 40,2 & 33,3 & 1,0 \\
\hline
\end{tabular}

Tabela 4 - Avaliação do índice de controle da incerteza (ICI)

Fonte: Elaborado pelo autor

A maioria das empresas (49\%) afirmam que algumas vezes os funcionários se sentem tensos ou nervosos e que alguma vez ou na maioria das vezes os funcionários preferem que as tarefas sejam bem estruturadas, com $73,5 \%$. Além disso, $77,5 \%$ das empresas afirmaram que alguma vez ou na maioria das vezes grande parte do trabalho é bem planejada e que as reuniões são frequentemente planejadas com antecedência $(73,5 \%)$.

O quinto grupo de questões para avaliação da cultura é a visão de longo ou curto prazo (IVI), que indica quando uma organização se baseia em tradições relativas a acontecimentos passados e presentes ou sobre os benefícios do que é desejável ao futuro. No longo prazo, os valores serão orientados para o futuro, como poupanças e persistência. Por outro lado, numa visão de curto prazo, os valores têm uma orientação para o passado e o presente, aceitando como verdade a tradição e o cumprimento das obrigações sociais.

Na tabela 5 é possível verificar os principais resultados das questões referentes a visão de longo e curto prazo.

Todos os respondentes afirmam que devem ser produzidos resultados rápidos pelo menos numa minoria das vezes. A lealdade pessoal varia de acordo com a necessidade do negócio em algumas vezes e na maioria das vezes $(73,6 \%)$.

\begin{tabular}{|c|c|c|c|c|c|c|}
\hline 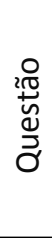 & 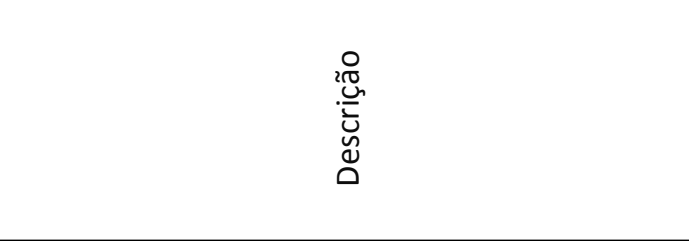 & $\begin{array}{l}\text { J } \\
\stackrel{5}{z} \\
\end{array}$ & 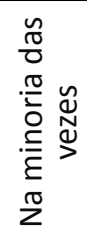 & 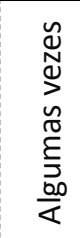 & 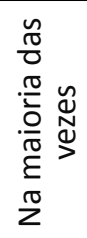 & 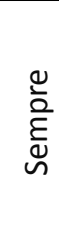 \\
\hline 17 & $\begin{array}{l}\text { Devem produzir resultados rápidos, o foco } \\
\text { está na geração de lucros do ano. }\end{array}$ & 0,0 & 15,7 & 41,2 & 41,2 & 2,0 \\
\hline 18 & $\begin{array}{l}\text { Lealdades pessoais variam de acordo } \\
\text { com as necessidades do negócio. }\end{array}$ & 3,9 & 20,6 & 41,2 & 32,4 & 2,0 \\
\hline 19 & $\begin{array}{l}\text { Principais Valores são liberdade e a } \\
\text { valorização das conquistas. }\end{array}$ & 0,0 & 22,5 & 44,1 & 28,4 & 4,9 \\
\hline 20 & O pensamento é na maioria das vezes analítico. & 0,0 & 14,7 & 46,1 & 38,2 & 1,0 \\
\hline
\end{tabular}

Tabela 5 - Avaliação do índice de visão de longo ou curto prazo (IVI)

Fonte: Elaborado pelo autor 
Além disso, algumas vezes e na maioria das vezes, os principais valores da empresa são liberdade e valorização das conquistas, com $72,5 \%$ dos respondentes. Por fim, o pensamento da maioria das empresas respondentes é alguma vez ou na maioria das vezes $(84,3 \%)$ analítico.

\subsection{Análise de correspondências múltiplas}

A análise de correspondência permitiu a melhor visualização, das associações existentes entre as variáveis de cultura organizacional e os dados gerais da empresa, como porte e nacionalidade. A figura 4 representa as dimensões avaliadas, na qual é possível identificar quatro grupos com associações. O primeiro grupo (1) refere-se às empresas nas quais os funcionários nunca escondem suas emoções; se sentem sempre tensos ou nervosos; nunca usam individualismo e egoísmo em suas relações profissionais e possuem liberdade para questionar ordens de seus dirigentes.

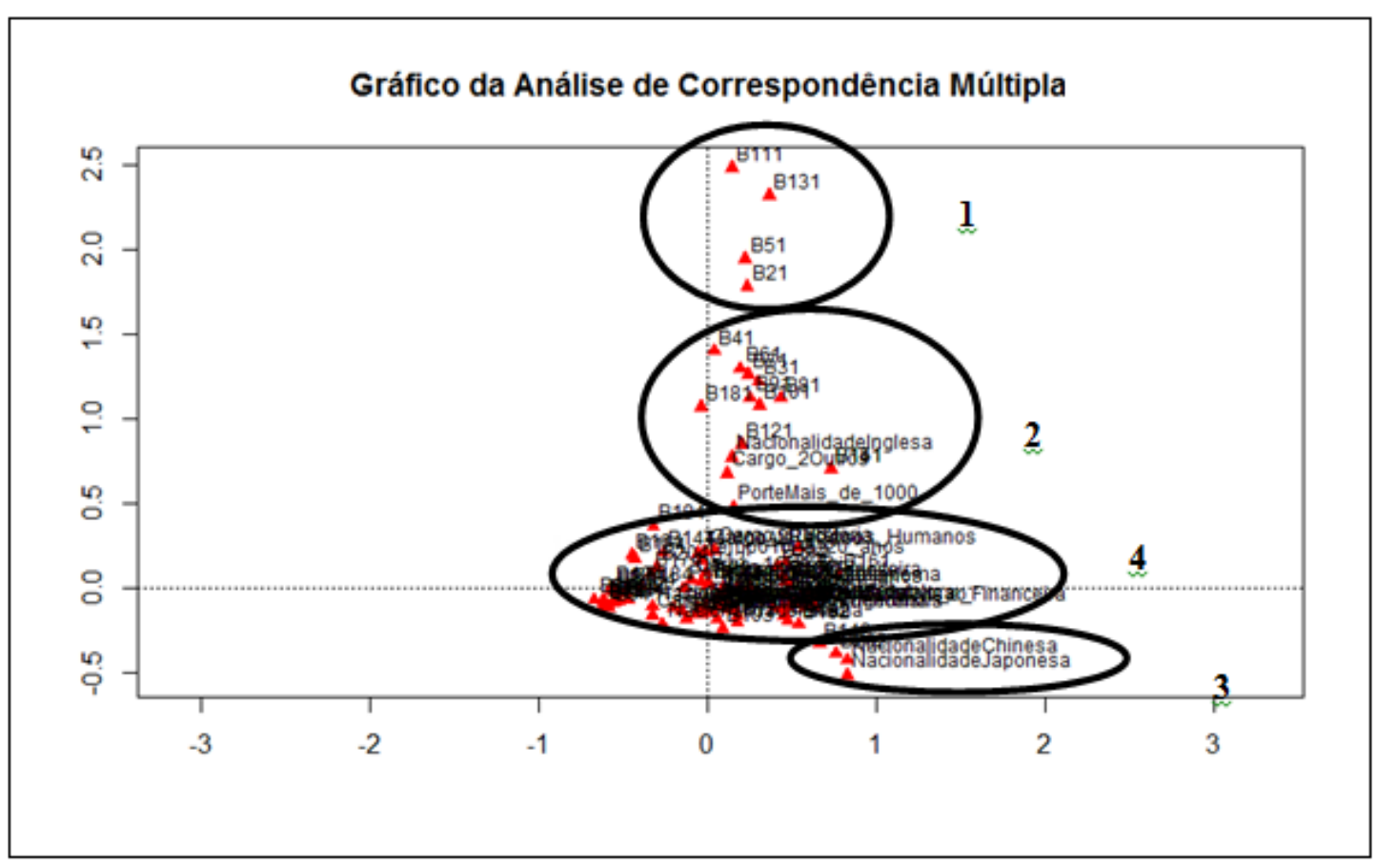

Figura 4 - Correspondência entre dados gerais das empresas e a cultura Fonte: Elaborado pelo autor

O segundo grupo (2) é caracterizado por empresas em que as posições mais elevadas hierarquicamente nunca têm privilégios diferentes daqueles com posições mais baixas; o indivíduo nunca é mais valorizado que o grupo; os interesses individuais nunca sobrepõe os coletivos; os funcionários podem falar diretamente com seus diretores; a maioria dos funcionários nunca se caracterizam por indelicadeza; as relações de competição profissional nunca são percebidas; as lealdades pessoais nunca variam de acordo com as necessidades do negócio; existem várias mulheres em cargos gerenciais; não ocorre uma divisão de papéis entre homens e mulheres; são empresas de nacionalidade inglesa e com porte de mais de 500 funcionários. 0 terceiro grupo (3) representa empresas de nacionalidade chinesa e japonesa para as quais ocorre uma divisão clara 
dos papéis entre homens e mulheres e o pensamento, na minoria das vezes tem uma característica analítica. $O$ último grupo (4) não é possível distinguir no gráfico, pois as empresas possuem características muito próximas.

\section{RESULTADOS E CONSIDERAÇÕES FINAIS}

Nessa pesquisa foi realizada a aplicação de um Survey no setor de cosmético brasileiro, o que permitiu o entendimento da percepção gerencial de 102 empresas do setor sobre o tema Cultura Organizacional. Como resultados gerais foram identificados nessas empresas que as pessoas têm pelo menos alguma influência baseada primeiramente no cargo que elas ocupam, alto nível de individualismo apontando para a priorização dos direitos individuais, num ambiente no qual os participantes tendem a formar um maior número de relações fora do ambiente de trabalho, são reforçados valores tradicionalmente masculinos de realização, como o controle e o poder, existe baixa tolerância à ambiguidade, havendo uma orientação para normas, regras ou controles, reduzindo o nível de incerteza, sendo necessária a produção de resultados rápidos. Tratam-se ainda de ambientes nos quais a lealdade pessoal varia de acordo com a necessidade do negócio.

Com relação à análise de correspondência, verifica-se uma associação entre empresas de nacionalidade inglesa e com porte de mais de 500 funcionários são as que posições mais elevadas hierarquicamente nunca têm privilégios diferenciados; o indivíduo nunca é mais valorizado que o grupo; o interesse individual nunca sobrepõe os coletivos; os funcionários tem acesso aos diretores; nunca ocorrem atos de indelicadeza; não são percebidas relações de competição profissional; as lealdades pessoais não variam de acordo com as necessidades do negócio; existem várias mulheres em cargos gerenciais; não ocorre uma divisão de papéis entre homens e mulheres. Empresas de nacionalidade chinesa e japonesa apresentam associação com empresas nas quais ocorre uma divisão clara dos papéis entre homens e mulheres e o pensamento, quase nunca se apresenta analítico.

Nesse estudo, foi possível identificar importantes elementos da cultura, existentes no setor de cosmético brasileiro, não esgotando as possibilidades de análise, que podem ser aprofundadas e comparadas com os resultados de outras abordagens, como sugestão ara futuras pesquisas no setor. 


\section{REFERÊNCIAS}

ALCÂNTARA, V. C. et al. Mensuração da Cultura Organizacional: Uma análise quantitativa - comparativa. Revista Eletrônica de Ciência Administrativa - RECADM, Campo Largo, v. 8, n. 2, p. 213-232, 2010. Disponível em: <http://revistas.facecla.com.br/index.php/recadm/article/view/621/493 $>$. Acesso em: 30 nov. 2012.

ALCÂNTARA, V. C., REIS, P.C., MENESES, R. S. S. Identificação de clusters internacionais com base nas dimensões culturais de Hofstede. Revista de Administração da UFSM. Santa Maria - RS, v. 5, n. 2, p. 204221, MAl./AGO. 2012. <http://cascavel.ufsm.br/revistas/ojs-2.2.2/index.php/reaufsm/article/view/2451/ pdf>

Acesso em: 30 nov. 2013.

BELMONTE, V. A. B., FREITAS, W. R. S. Identificação de clusters internacionais com base nas dimensões culturais de Hofstede. Revista de Administração da UFSM. Santa Maria - RS, v. 6, n. 1, p. 71-90, JAN./MAR. 2013. < http://cascavel.ufsm.br/revistas/ojs-2.2.2/index.php/reaufsm/article/view/2465/pdf $>$ Acesso em: 26 nov. 2014.

CAMERON, K. S.; QUINN, R. E. Diagnosing and changing organizational culture. 2. ed. San Francisco: Jossey-Bass, 2006. $242 \mathrm{p}$.

CHIAVENATO, I. Gestão de pessoas. 4. ed. Rio de Janeiro: Elsevier, 2014. 579 p.

CRANE, A. Rhetoric and reality in the greening of organizational culture. Greener Management International, Sheffield, v. 11, n. 12, p. 49-62, 1995.

DENISON, D., HOOIJBERG R., LANE N., LIEF C. Leading Culture Change in Global Organizations: Aligning Culture and Strategy. San Francisco: Jossey-Bass, 2012.

FISCHER, R. M. Poder e cultura em organizações penitenciárias. 1989. Tese (Livre Docência em Administração) - Faculdade de Economia e Administração, Universidade de São Paulo, 1989.

FLEURY, M. T.; FLEURY, A. Aprendizagem e Inovação Organizacional. 2 ed. São Paulo: Atlas, 1997.

HOFSTEDE, G. Culture and organizations. International Studies of Management and Organizations, New 
York, v. 10, n. 4, p. 15-41, 1981.

HOFSTEDE, G. Culture's consequences: international differences in work-related values. Beverly Hills: Sage, 1980. $475 \mathrm{p}$.

HOFSTEDE, G. Identifying organizational subcultures: An empirical approach. Journal of Management Studies, v. 35, n.1; p. 1-12, 1998.

HOFSTEDE, G. Cultures and Organizations: software of the mind. Maidenhead: McGraw-Hill, 1991. 279 p.

HOMBURG, C.; PFLESSER, C. A multiple-layer model of market-oriented organizational culture: measurement issues and performance outcomes. Journal of Marketing Research, Chicago, v. 37, n. 4, p. 449-462, nov. 2000.

LINNENLUECKE, M. K.; GRIFFITHS, A. Corporate sustainability and organizational culture. Journal of World Business, v. 45, n. 4, p. 357-366, 2010.

LINNENLUECKE, M. K.; RUSSELL, S. V.; GRIFFITHS, A. Subcultures and sustainability practices: the impact on understanding corporate sustainability. Business Strategy and the Environment, Queensland, v. 18, n. 7, p. 432-452, 2009.

MILANI, B., RIGHI, M. B., CERETTA, P. S. Práticas de Sustentabilidade, Governança Corporativa e Responsabilidade Social afetam o Risco e o Retorno dos Investimentos - Revista de Administração da UFSM. Santa Maria - RS, v. 5, Edição Especial, p. 667-682, DEZ. 2012. < http://cascavel.ufsm.br/revistas/ ojs-2.2.2/index.php/reaufsm/article/view/6946/pdf> Acesso em: 01 nov. 2014.

MOTTA, F. P.; CALDAS, M. P. Cultura organizacional e cultura brasileira. São Paulo: Atlas, 1997. 325 p.

OUCHI, W. G.; PRICE, R. L. Hierarchies, clans, and theory Z: A new perspective on organization development. Organizational Dynamics, v. 21, n. 4, p. 62, 1993.

QUINN, R. E. Change the World: how ordinary people can accomplish extraordinary results. San Francisco: Jossey-Bass, 2000. $304 \mathrm{p}$. 
RAMOS, A. G.; CARDOSO, M. A nova ciência das organizações: uma reconceituação da riqueza das nações. 2. ed. Rio de Janeiro: Fundação Getúlio Vargas, 1989. 209 p.

ROBBINS, S.; JUDGE, T. Organizational behavior. 15a. ed. U. S. A.: Prentice Hall, Cloth, 720 pp, 2013.

SANTOS, N. M. B. F. Cultura organizacional e desempenho: pesquisa, teoria e aplicação. Lorena: Stiliano, 2000.

SCHEIN, E. H. Organizational Culture and Leadership: a dynamic view. San Francisco: Jossey-Bass, 1992. $380 \mathrm{p}$.

SCHEIN, E. H. Organizational Culture and Leadership. 3. ed. San Francisco: Jossey-Bass, 2004. 448 p.

SCHWARTZ, H.; DAVIS, S. M. Matching corporate culture and business strategy. Organizational Dynamics, v. 10 , n. 1 , p. 30-48, 1981.

SILVA, L. M. T.; MEDEIROS, C. A.; ENDERS, W. T. Avaliação da Cultura Organizacional: um contraponto entre as abordagens quantitativas e qualitativas. INTERFACE - Revista do Centro de Ciências Sociais Aplicadas, Natal, v. 8, n. 2, p. 124-140, 2011. Disponível em: <http://ccsa.ufrn.br/ojs/index.php/interface/article/ view/463/430>. Acesso em: 05 dez. 2012.

SMIRCICH, L. Concepts of culture organizational analysis. Administrative Science Quarterly, v.28, n.3, p.339-358, sep. 1983.

SOUZA, E. L. P. Clima e cultura organizacionais: como se manifestam e como se manejam. São Paulo: Edgar Blücher, 1978. p. 92.

TRICE, H. M., BEYER, J. M. Studying Organizational Cultures Through Rites and Ceremonials. Academy of Management Review, New York, vol. 9, n. 4, p. 653-669, oct.1984.

Alguns Websites usados como referencia:

http://www.cosmeticsonline.com.br

http://www.cosmeticsandtoiletries.com/

http://www.abihpec.org.br/

Rev. AdM. UFSM, SANTA MARIA, v. 11, NúMERo 5, P. 1305-1323, 2019 